\author{
CHRISSY LAURENTZEN \\ Radboud Universiteit Nijmegen, Niederlande \\ chrissy.laurentzen@student.ru.nl \\ SABINE JENTGES \\ Radboud Universiteit Nijmegen, Niederlande \\ s.jentges@let.ru.nl
}

\title{
Da oder weil, daher oder deswegen? / Weil es nicht zielsprachenadäquat ist...? Deswegen! Zielsprachenadäquate Ausdrücke von Kausalität in L1- und L2-Texten im Deutschen
}

\section{Da or weil, daher or deswegen? / Because it is not adequate for the target language...? That's why! Target language adequacy of causal adverbs in L1- and L2-Texts in German}

\begin{abstract}
Grammar books for L2-learners mostly follow grammatical themes, e.g. sentence verb - conjunctions. However, when a L2-learner composes a text, he will probably focus more on the function he wants to express than the form he wants to use. In this article, functionality versus grammatical categories will be discussed in order to answer the question, whether and - if so - how German and Dutch students differ in their way of expressing causality in academic writing. An analysis of German Bachelor's and Master's theses written by German and Dutch students showed differences between these groups in their use of subcategories (word classes) and specific words for expressing causality. The absence of a functional structure and specific information about the use of causal connectives in grammar books can therefore be regarded as an important cause for these findings. Especially for advanced L2-learners of German it seems recommendable to organize grammar books to put a greater focus on function (rather than form).

KEYWORDS: German as a foreign language (GFL), causality, target language adequacy, grammar books, educational material, advanced L2-learners, functionality, grammatical categories, Netherlands.
\end{abstract}


SCHLÜSSELWORTE: Deutsch als Fremdsprache (DAF), Kausalität, Zielsprachenadäquatheit, Grammatiken, Lehr- und Lernmaterialien, fortgeschrittene L2-Lerner, Funktionalität, grammatische Kategorie, die Niederlande.

\section{ZIELSPRACHENADÄQUATE TEXTE IN DER L2}

Texte in einer Fremdsprache auf eine Weise zu verfassen, dass sie als zielsprachenadäquat gelten können, ist eine äußerst anspruchsvolle Aufgabe, die auch bei sehr fortgeschrittenen Lernenden häufig misslingt. Bei fremdsprachlichen Textproduktionen auf den höchsten GeR-Niveaus C1/C2 - beispielsweise im Rahmen eines Germanistikstudiums in den Niederlanden ${ }^{1}$ - ist immer wieder festzustellen, dass Texte zwar auf morphologischer und syntaktischer Ebene nahezu fehlerlos sind, aber dennoch nicht als zielsprachenadäquat eingestuft werden können (Jentges 2011: 237ff.; Jentges 2012: 235f. sowie Jentges 2016: 201f.). Textproduktionen (nicht nur) in einer Fremdsprache erfordern nicht allein die Beherrschung der Zielsprache auf morphosyntaktischer Ebene, sondern auch im Bereich der Semantik. Darüber hinaus sind Kenntnisse zielsprachenspezifischer Textmuster und -konventionen erforderlich. Dieses, nicht immer sprachenübergreifende Textsortenwissen kann sich auf die inhaltliche und formale Gestaltung eines Textes beziehen, auf sprachenspezifische informationsstrukturelle Muster, die z.B. einhergehen mit einer angemessenen Realisation von Kohärenz und Kohäsion oder auch mit sprachenspezifischen Mustern, wie bestimmte Aussageabsichten sprachlich realisiert werden.

Im vorliegenden Beitrag steht die Realisation von Kausalität in der Zielsprache Deutsch im Mittelpunkt, die L2-Lernenden nicht immer zielsprachenadäquat gelingt. Gründe hierfür können beispielsweise Interferenz - also spezifische Formunterschiede oder Bedeutungsnuancen im Vergleich zu der jeweiligen L1 - sein (Einig \& Menne-El-Sawy 2012: 387ff), die Tatsache, dass es in anderen Kulturen unterschiedliche nennenswerte Gründe gibt (Zufferey, Mak \& Sanders 2015: 26) und die, dass jede Textsorte Konnektoren unterschiedlich häufig benutzt. Smith and Frawley (1983: 369ff) haben für das Englische gezeigt, dass wissenschaftliche Texte relativ wenige kausale Konjunktionen benutzen, dafür aber ein abwechslungsreicheres und komplexeres Vokabular einsetzen. Der Gebrauch von Wissenschaftssprache verlangt eine hohe sprachliche Kompetenz, für die Fremdsprachenlerner ${ }^{2}$ nicht nur eine wissenschaftliche Schreibkompetenz entwickeln, sondern auch die sprachspezifische Aus-

1 An der Radboud Universiteit in Nijmegen ist das Abschlussniveau am Ende des dreijährigen Bachelorstudiums Deutsche Sprache und Kultur C2 (nach GeR), sowohl im Bereich der rezeptiven als auch produktiven Sprachbeherrschung.

${ }^{2}$ Zugunsten der Lesbarkeit sind die Personennennungen in diesem Beitrag generisch, es sind also stets beide Geschlechter gemeint. 
übung dieser Kompetenz erwerben müssen (Mächler 2012: 537; Mohammadi 2009: 140f).

Selbst wenn Sprachen sehr nah verwandt sind, wie das Deutsche und das Niederländische, gibt es Formunterschiede, Bedeutungsunterschiede und auch Unterschiede in der Häufigkeit der Verwendung sprachlicher Ausdrucksmittel. Für den Bereich der Kausalität hat Kántor-Faragó (2006) für die rückwärts implizierenden Kausalkonjunktionen einen Vergleich zwischen den nahverwandten Sprachen Deutsch und Niederländisch durchgeführt, indem sie den Gebrauch der deutschen kausalen Konnektoren ,weil', ,denn' und ,da' und ihrer niederländischen Übersetzungen sowie der niederländischen kausalen Konnektoren ,omdat', ,want' und ,aangezien' und ihrer deutschen Übersetzungen in literarischen Korpora analysiert hat. In der Untersuchung wird deutlich, dass die deutschen und niederländischen Konjunktionen nicht miteinander gleichgesetzt werden können, sie unterscheiden sich syntaktisch, aber v.a. auch semantisch (Kántor-Faragó 2006: 87f). Aus fremdsprachendidaktischer Perspektive mag es sich dabei um nur als marginal zu bezeichnende Unterschiede handeln, die im regulären Fremdsprachenunterricht auf den A- und B-Niveaus (nach GeR) zu komplex bzw. zu wenig kommunikativ relevant sind, um thematisiert zu werden. Auch im Deutschunterricht in den Niederlanden werden solche Unterschiede im Detail üblicherweise nicht behandelt. Handelt es sich jedoch um weit fortgeschrittene Germanistikstudierende, die ihren Bachelor- oder Masterabschluss machen, sind diese kleinen, aber feinen Unterschiede sehr wohl von Bedeutung; sie tragen dazu bei, dass ihre Abschlussarbeiten nicht nur als nahezu fehlerfrei, sondern auch als zielsprachenadäquat eingestuft werden können.

Relevant ist in diesem Kontext auch, dass die Vermittlung bestimmter Redeabsichten im Fremdsprachenunterricht häufig nach morphosyntaktischen Aspekten erfolgt, jedenfalls dann, wenn hierfür Grammatiken zu Rate gezogen werden. Die gängigen Lernergrammatiken (wie z.B. Engel 1988; Helbig \& Buscha 1991; Kars \& Häussermann 1992 und die für den Deutschunterricht in den Niederlanden bekannte und verbreitetste Grammatik von Ten Cate, Lodder \& Kootte 2013) untergliedern ihre Kapitel nach grammatischen Kategorien, die Funktionen der jeweiligen Wortart präsentieren. Davon ausgehend, dass Sprache als Mittel zur Kommunikation dient, erscheint diese Darstellungsweise wenig funktional. Eine Grammatik, die im Gegensatz hierzu anders, nämlich semantisch-funktional, organisiert ist, ist die Grammatik in Feldern (Buscha et al. 1998). Diese Grammatik bespricht in den einzelnen Kapiteln kommunikative Funktionen, wie beispielsweise Kausalität. So werden hier im Kapitel Feld der Begründung verschiedene Möglichkeiten illustriert, wie Kausalität im Deutschen ausge- 
drückt werden kann. L2-Lernern wird somit ermöglicht, von ihrer kommunikativen Absicht ausgehen zu können und diese nicht vorab bereits einer bestimmten Wortart (wie etwa Konjunktion, Präposition) zuordnen zu müssen. Ruth Albert (2008: 106f.) ist sicher zuzustimmen, dass eine solche Darstellung für Anfänger zu komplex und unübersichtlich sein dürfte, da zu viele Möglichkeiten auf einmal dargestellt werden könnten bzw. müssten, doch gerade für fortgeschrittene Lernende, wie die beschriebene Gruppe niederländischer Germanistikstudierender, dürfte ein solcher funktionaler Ansatz ideal sein. Eine Darstellung, die zeigt, wie eine bestimmte kommunikative Absicht ausgedrückt werden kann und wie dies üblicherweise auch von L1-Sprechern bzw. -Schreibern gemacht wird, unterstützt fortgeschrittene L2-Lerner dabei, sich einen Überblick über passende zielsprachige Sprachmittel zu verschaffen und ein zielsprachenadäquat(er)es Niveau im Bereich Frequenz und Variation zu erreichen.

Ausgehend von diesen Überlegungen, hat die diesem Beitrag zugrundeliegende Studie (Laurentzen 2016) erhoben, ob und falls wie sich deutsche und niederländische Studierende bezüglich der sprachlichen Mittel, die sie im Deutschen einsetzen, um Kausalität in wissenschaftlichen Texten auszudrücken, voneinander unterscheiden. Erwartet wird, dass sehr fortgeschrittene niederländische Studierende im Vergleich zu Studierenden mit Deutsch als L1 andere kausale Verknüpfungsmittel bzw. diese mit unterschiedlicher Frequenz verwenden.

\section{AUSDRUCKSMITTEL FÜR KAUSALITÄT}

Die deutsche Sprache verfügt über eine große Anzahl kausaler Verknüpfungsmittel. Neben den Subjunktionen ,weil', ,da', ,zumal', ,umso mehr als' zur Bildung von kausalen Nebensätzen und der Konjunktion ,denn' sowie den Partikeln ,nämlich' und ,doch' zur Bildung von Hauptsätzen (Buscha et al. 1998: 56ff), können Nebensätze bzw. Zweitsätze auch ohne Verbindungswort vorkommen; dann wird Kausalität auf semantischer Ebene realisiert (Buscha et al. 1998: 63; Breindl \& Waßner 2006: 55ff). Ebenso können auch Partizipialkonstruktionen Kausalität ausdrücken (mit Partizip I: ,Das Wetter befürchtend, nahm sie einen Regenschirm mit.' oder mit Partizip II: ,Von dem Gewitter gewarnt, blieb sie noch etwas länger zu Hause.'), wie auch Präpositionen. Die laut Buscha et al. (1998: 66) am häufigsten verwendeten kausalen Präpositionen sind ,wegen' und ,aufgrund'; aber auch ,angesichts', ,anhand', ,anlässlich', ,dank', ,halber', ,infolge', ,kraft', ,mangels', ,vermöge', , um... willen', ,zufolge' und ,zuliebe' gehören zu dieser Kategorie. Des Weiteren gibt es auch Präpositionen, die neben anderen Funktio- 
nen eine kausale Bedeutung haben können (z.B. ,aus', , vor', , auf', ,durch' und , von'). ${ }^{3}$

Auch lexikalische Sprachmittel können Kausalität ausdrücken, hierzu gehören u.a. Verben, wie ,auslösen', ,bewirken', ,(mit sich) bringen', ,hervorrufen', ,verursachen', ,führen $\mathrm{zu}^{\prime}$ und Substantive, wie ,Anlass', ,Grund', ,Motiv' und ,Ursache' (Buscha et al. 1998: 74f). Dies gilt auch für Komposita von Substantiven oder Adjektiven, bei denen das Erstglied gewöhnlich den Grund ausdrückt (z.B. ,Bissverletzung': eine Verletzung, die durch einen Biss entstanden ist). Schließlich können auch Adverbien benutzt werden, um Kausalität auszudrücken, z.B. Zusammensetzungen mit ,-halber' (Buscha et al. 1998: 73) oder auch Adverbien wie ,deshalb', ,daher', ,darum', ,deswegen' und ,dadurch' (Duden 2009: 1086; Ten Cate et al. 2013: 393; Blühdorn 2006: 259).

\section{ANALYSE KAUSALER AUSDRUCKSMITTEL}

Der hier vorgestellten Analyse liegt ein Korpus (Laurentzen 2016) von deutschsprachigen Bachelor- und Masterarbeiten aus dem geisteswissenschaftlichen Bereich zugrunde, insg. bestehend aus 301.666 Wörtern bzw. 987 Druckseiten. Der Korpusanteil der Verfasser mit Deutsch als L14 umfasste 150.693 Wörter bzw. 490 Druckseiten und diente als Vergleichsmaterial, um festzustellen, wie eine zielsprachenadäquate Verwendung von Kausalität in L1-Texten realisiert wird. Der Korpusanteil der von niederländischen Studierenden auf Deutsch geschriebenen Arbeiten umfasste 150.973 Wörter bzw. 497 Druckseiten. Die Sprachkenntnisse der niederländischen Studierenden im Deutschen sind nach GeR auf Niveau C2 zu beschreiben (vgl. Fußnote 1), alle haben Niederländisch als L1 und Deutsch als Fremdsprache als L3 oder L4 (nach Englisch und ggf. Französisch) gelernt.

Bei der Analyse des Korpus wurden die auf kausaler Ebene verwendeten Ausdrücke kategorisiert. ${ }^{5}$ Die Kategorienbildung für die Analyse orientierte sich an der Darstellung möglicher kausaler Ausdrucksmittel in der Gramma-

${ }^{3}$ Die hier erfolgte Darstellung orientiert sich an Buscha et al. (1998: 69f), hat aber keinen Anspruch auf Vollständigkeit.

${ }^{4}$ Es handelt sich um Studierende, deren einzige L1 Deutsch ist und die an den engsten Partneruniversitäten (Westfälische Wilhelms-Universität Münster sowie Universität DuisburgEssen) der Abteilung Deutsche Sprache und Kultur/Niederlande-Deutschland-Studien der Radboud Universiteit im Bereich Germanistik ihre Studienabschlussarbeit auf Deutsch geschrieben haben.

${ }^{5}$ Kausale Ausdrucksmittel, die in einem direkten Zitat erschienen, wurden bei der Analyse nicht berücksichtigt. 
tik in Feldern (Buscha et al. 1998) und wurde mithilfe der Duden Grammatik (Duden, 2009), die ebenfalls eine umfangreiche Übersicht zum Ausdruck von Kausalität enthält, abgeglichen und ergänzt. Die Analysekategorien ermöglichten sowohl eine quantitative (Wie häufig wurden die Mittel von den jeweiligen Verfassern benutzt und wie verhält sich dies zueinander?) als auch eine qualitative Auswertung (Welche Mittel wurden benutzt und wie verhält sich dies zueinander?). Da die Analyse per Hand durchgeführt werden musste, konnten auch eventuelle auffällige Unterschiede innerhalb der Kategorien hervorgehoben und besprochen werden.

Die Sprachmittel, die benutzt werden können, um Kausalität auszudrücken, können in morphosyntaktische und lexikalische Mittel untergliedert werden (vgl. Kapitel 2). ${ }^{6}$ Folgende Übersicht nach Buscha et al. (1998) und Duden (2009) zeigt die aufgestellten Kategorien.

1. Grammatische Sprachmittel
A. Nebensatz/Hauptsatz
B. Partizipialkonstruktion
C. (Kausale) Präposition

2. Lexikalische Sprachmittel
A. Nomen
B. Verb
C. Adverb
D. Partikel

Die Analysekategorien zeigten einen Unterschied zwischen grammatischen und lexikalischen Konnektoren. Es stellte sich heraus, dass die deutschen Studierenden diese Mittel ungefähr gleichhäufig einsetzen: 51,9\% der benutzten kausalen Ausdrucksmittel waren grammatische Mittel, während 47,9\% der benutzten kausalen Ausdrücke lexikalische Mittel waren. ${ }^{7}$ Innerhalb der Kategorie der grammatischen Konnektoren stellt die Nebensatz/Hauptsatz-Kategorie mit 359 Ausdrücken (33,9\% der gesamten genutzten kausalen Ausdrücke) die größte Subkategorie dar. Präpositionen folgen mit

${ }^{6}$ In Kapitel 2 wurden ebenfalls Hauptsätze ohne Verbindungswort und Komposita als kausale Ausdrucksmittel genannt. Die Beurteilung dieser Mittel bleibt selbst bei mehreren Ratern äußerst subjektiv, denn die Anwesenheit einer kausalen Bedeutung kann hier nur durch Interpretation erfolgen. Es ist daher aus praktischen und Objektivitätsgründen entschieden worden, diese beiden Kategorien nicht weiter zu berücksichtigen. Es sind somit bestimmte Realisierungen möglicher kausaler Beziehungen nicht in der Analyse berücksichtigt.

7 Die übrigen 0,2\% sind Ausdrücke, die nicht nach den Analysekategorien eingeteilt werden konnten. Es handelt sich hier um zwei Adjektive: ,hauptverantwortlich' und ,ursächlich'. 
17,1\% (181 Ausdrücke) der Gesamtzahl kausaler Ausdrücke. Partizipialkonstruktionen wurden nur zehn Mal verwendet (0,9\% der gesamten Zahl der kausalen Ausdrücke) und sind damit die kleinste Kategorie innerhalb der von den deutschen Studierenden benutzten grammatischen Ausdrucksmittel. Lexikalische Mittel sind im Gegensatz dazu etwas proportionaler eingesetzt worden. Am häufigsten wurden Adverbien benutzt (205, 19,3\%). Die Verben $(157,14,8 \%)$ und Nomina $(142,13,4 \%)$ nehmen die zweite und dritte Position innerhalb der lexikalischen Mittel ein. Partikeln wurden von den deutschen Studierenden mit nur 0,4\% am wenigsten häufig in ihren Texten benutzt.

Niederländische Studierenden nutzen grammatische Mittel (554, 53,7\%) nur etwas häufiger als lexikalische Mittel (476, 46,1\%). ${ }^{8}$ Innerhalb der Kategorie der grammatischen Mittel wurde mit 461 Ausdrücken (44,7\% der gesamten Anzahl der kausalen Ausdrücke im L2-Korpus) die Nebensatz/ Hauptsatz-Kategorie am häufigsten verwendet. Die Präpositionen und v.a. die Partizipialkonstruktionen wurden vergleichsweise selten eingesetzt: Die niederländischen Studierenden benutzten 91 Präpositionen $(8,8 \%)$ und nur zwei Partizipialkonstruktionen (0,2\%). Die Partizipialkonstruktionen stellen somit die am wenigsten realisierte Kategorie im L2-Korpus dar. Innerhalb der Kategorie der lexikalischen Mittel wurden die Adverbien von den niederländischen Studierenden am häufigsten benutzt (204, 19,8\%), es folgen Verben $(135,13,1 \%)$, Nomina $(87,8,4 \%)$ und Partikeln $(50,4,8 \%)$.

\subsection{Art der Konnektoren: Vergleich Deutsch als L1 oder L2}

Wie sich der Gebrauch der kausalen Konnektoren der beiden Studierendengruppen zueinander verhält, ist in Abbildung 1 (für das Verhältnis zwischen den grammatischen und lexikalischen Mitteln) und Abbildung 2 (für das Verhältnis der Arten der Konnektoren) illustriert. Es zeigt sich, dass die beiden Gruppen im Gebrauch der Hauptkategorien der grammatischen und lexikalischen Mittel übereinstimmten. Die niederländischen Studierenden benutzten mit 53,7\% nur etwas häufiger grammatische Mittel und mit $46,1 \%$ etwas weniger häufig lexikalische Mittel als die deutschen Studierenden, die zu 51,9\% grammatische Mittel und zu 47,9\% lexikalische Mittel einsetzten. Dass die Unterschiede marginal sind, bestätigt auch die statistische Analyse: Es gibt keinen Effekt der L1 auf die Hauptkategorien der verwendeten Konnektoren $\left(\chi^{2}(2)=0,681, p=0,71\right)$.

\footnotetext{
8 Die übrigen $0,2 \%$ bestehen aus anderen Ausdrucksmitteln. Es handelt sich hier um ein Adjektiv, nämlich ,abhängig', und einen nicht-zielsprachenadäquaten Ausdruck.
} 


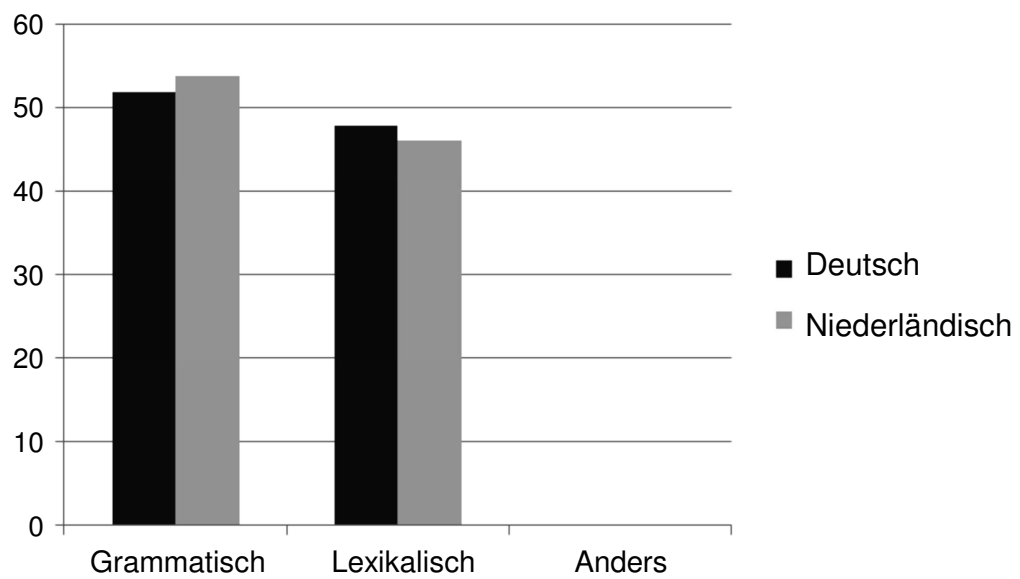

Abbildung 1. Grafische Darstellung des Gebrauchs der Hauptkategorien der deutschen und niederländischen Studierenden in Prozentzahlen

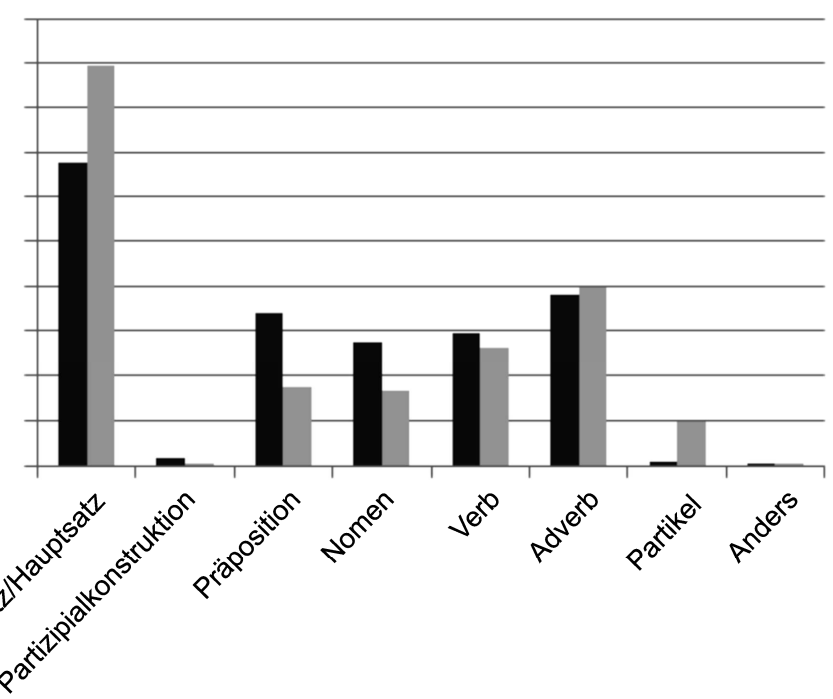

Deutsch

Niederländisch

Abbildung 2. Grafische Darstellung der Unterschiede zwischen den deutschen und niederländischen Studierenden im Gebrauch der Arten der Subkategorien der Konnektoren in Prozentzahlen

Die statistische Analyse zeigt allerdings einen Effekt der L1 auf die Verwendung der Subkategorien der Konnektoren $\left(\chi^{2}(7)=102,797, p<0,001\right)$. Abbildung 2 illustriert Übereinstimmungen und Unterschiede zwischen diesen Kategorien. Die beiden Studierendengruppen stimmten im Gebrauch 
der Adverbien (Niederländisch: 19,8\%, Deutsch: 19,3\%) und der Verben (Niederländisch: 13,1\%, Deutsch: 14,8\%) weitestgehend überein. Dies bestätigt auch die statistische Analyse bezüglich des Gebrauchs dieser Wortarten, es gibt keine signifikanten Unterschiede $(p>0,05)$. Eine weitere Übereinstimmung ist, dass die Nebensatz-/Hauptsatz-Kategorie von beiden Gruppen am häufigsten verwendet wurde. Die niederländischen Studierenden benutzten diese Kategorie in fast der Hälfte der Fälle $(44,7 \%)$, in denen sie Kausalität ausdrücken möchten. Die deutschen Studierenden verwendeten diese Konstruktion seltener, nämlich in ungefähr einem Drittel der Fälle (33,9\%). Obwohl diese Subkategorie also von beiden Gruppen am häufigsten verwendet wurde, ist der Unterschied in der Frequenz des Gebrauches signifikant $(p<0,05)$.

Die Gruppen unterscheiden sich deutlich in der Kategorie, die sie am geringsten einsetzten. Während die Niederländer Partizipialkonstruktion $(0,2 \%)$ am wenigsten verwendeten, benutzten die Deutschen kaum Partikeln $(0,4 \%)$. Hierbei fällt zusätzlich auf, dass die Partizipialkonstruktionen von den deutschen Studierenden ebenfalls sehr wenig eingesetzt wurden $(0,9 \%)$, während die Partikeln von den niederländischen Studierenden im Vergleich $\mathrm{zu}$ den deutschen Studierenden häufiger eingesetzt wurden (4,8\%). Auch hier bestätigt die statistische Analyse, dass die Gruppen sich bezüglich des Gebrauchs dieser beiden Wortarten signifikant voneinander unterscheiden $(p<0,05)$. Die deutschen Studierenden verwendeten somit im Vergleich zu den niederländischen Studierenden signifikant mehr Partizipialkonstruktionen, während die niederländischen Studierenden signifikant öfter Partikeln benutzten.

Weitere Unterschiede zeigen sich im Gebrauch der Nomina und Präpositionen. Diese Kategorien wurden von den deutschen Studierenden häufiger verwendet als von den niederländischen Studierenden. Für Nomina waren dies 13,4\% bei den deutschen Studierenden im Vergleich zu 8,4\% bei den niederländischen Studierenden. Für die Präpositionen ist dieser Unterschied sogar noch etwas größer, mit 17,1\% für die deutschen Studierenden und $8,8 \%$ für die niederländischen Studierenden. Beide Unterschiede sind signifikant $(p<0,05)$.

Des Weiteren zeigt sich, dass die Verwendungsfrequenz kausaler Ausdrücke der deutschen Studierenden in den anderen Kategorien (Nomina, Verben, Präpositionen und Adverbien) relativ proportional verteilt ist: Diese Wortarten wurden durchschnittlich zu 13 bis 20\% verwendet. Bei den niederländischen Studierenden sieht man dagegen eine stärkere Präferenz für bestimmte Ausdrucksmittel: Partizipialkonstruktionen werden kaum benutzt (zu 0,2\%), Partikeln häufiger (zu 4,8\%), dann folgen Nomina und Präpositionen (zu 8,4 bzw. 8,8 \%), Verben (13,1\%) und Adverbien (19,8\%), und schließlich Neben- und Hauptsätze in fast der Hälfte aller Fälle $(44,7 \%)$. 


\subsection{Vergleich auf Wortniveau}

Die qualitative Analyse zeigt, dass es auf Wortniveau ebenfalls Unterschiede zwischen den niederländischen und den deutschen Studierenden gibt, die auf Basis der Zahlen pro Konnektorkategorie nicht ersichtlich geworden wären. Ein erster Unterschied wird beim Gebrauch der Neben- und Hauptsatzkonnektoren deutlich. Die statistische Auswertung bestätigt, dass es einen Effekt der L1 auf die Verwendung der Neben- und Hauptsatzkonnektoren gibt $\left(X^{2}(3)=198,579, p<0,001\right)$. Dieser Unterschied besteht zum einen darin, dass die deutschen Studierenden in drei Viertel der Fälle, in denen sie eine Konjunktion eingesetzt haben, , da' $(75,2 \%)$ verwendeten und damit eine deutliche Präferenz für diese Subjunktion zeigten. Dies ist signifikant öfter der Fall als bei den niederländischen Studierenden $(p<0,05)$. Diese Präferenz ist zudem nicht nur ersichtlich innerhalb der Klasse der Neben- und Hauptsatzkonnektoren, sondern auch für die kausalen Ausdrucksmittel im Allgemeinen: 25,5\% aller kausaler Ausdrücke im L1-Korpus werden mittels der Subjunktion ,da' gebildet. Die Konjunktion ,denn' wird mit 14,2\% (4,8\% aller kausaler Ausrücke) innerhalb der Klasse der Konjunktionen am zweithäufigsten verwendet, während die Subjunktion ,weil' mit 2,5\% (0,9\% aller kausaler Ausdrücke) von den deutschen Studierenden am seltensten verwendet wurde.

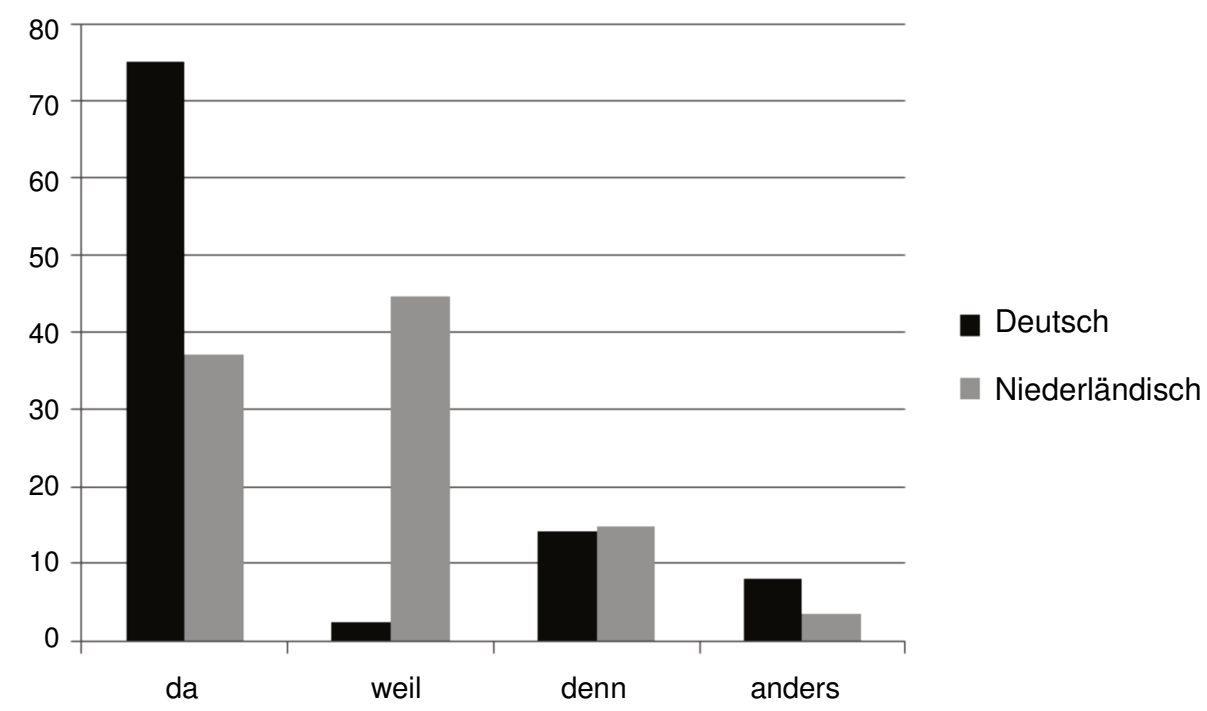

Abbildung 3. Grafische Darstellung der Unterschiede und Gemeinsamkeiten im Gebrauch der verschiedenen Neben- und Hauptsatzkonnektoren in Prozentzahlen 
Im L2-Korpus konnten deutliche Unterschiede zum L1-Korpus nachgewiesen werden, vgl. auch Abbildung 3. Die Subjunktion, die von den Niederländern am häufigsten verwendet wurde $(44,5 \%)$, ist ,weil'. Die niederländischen Studierenden benutzten diese Subjunktion signifikant öfter als die deutschen Studierenden $(p<0,05)$. Die von den Deutschen beliebte Subjunktion ,da' wird im Vergleich $\mathrm{zu}$,weil' etwas weniger häufig benutzt, nämlich in 37,5 \% der Fälle, in denen eine Konjunktion verwendet wurde, und wurde damit im Vergleich zu den deutschen Studierenden signifikant weniger häufig eingesetzt $(p<0,05)$. Der Gebrauch der Konjunktion ,denn' ist mit 14,9\% vergleichbar mit dem Gebrauch im L1-Korpus $(p>0,05$, kein signifikanter Unterschied). Die niederländischen Studierenden benutzten außerdem signifikant weniger häufig als die deutschen Studierenden ,ande-

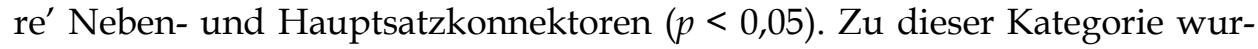
den z.B. ,zumal', ,so...dass' und ,umso mehr als' gezählt.

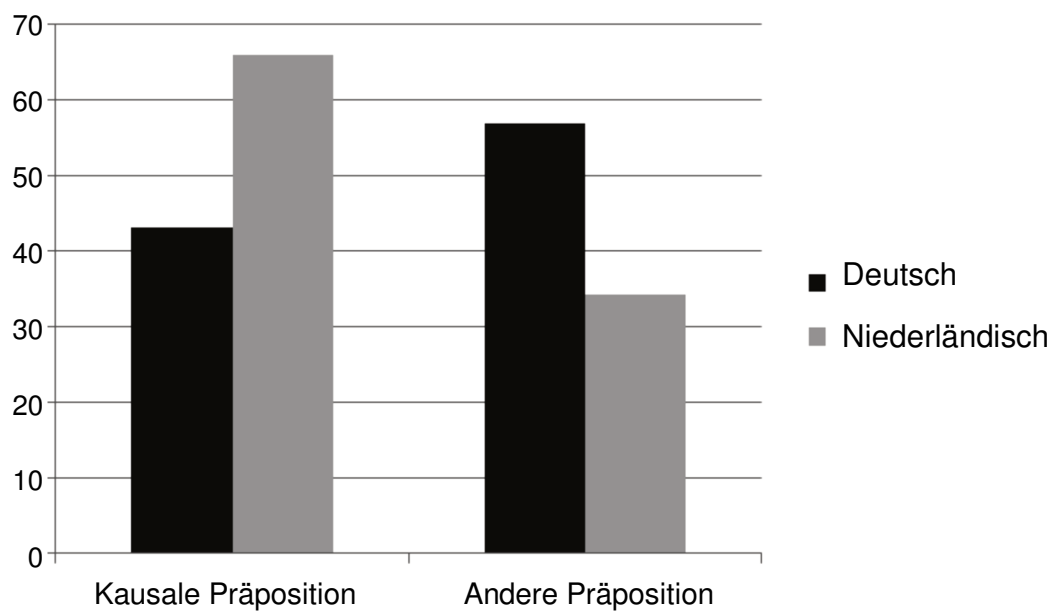

Abbildung 4. Grafische Darstellung der Unterschiede im Gebrauch der kausalen und anderen Präpositionen in Prozentzahlen

Unterschiede im Gebrauch zeigen sich auch bei Präpositionen: Es gibt einen Effekt der L1 auf die Verwendung der einzelnen Präpositionen $\left(\chi^{2}(1)\right.$ $=12,639, p<0,001)$. Innerhalb der Klasse der Präpositionen wird ein Unterschied zwischen kausalen Präpositionen und Präpositionen, die nicht zwingend kausale Bedeutung tragen, gemacht. Deutsche Studierende verwendeten etwas häufiger Präpositionen mit optional kausaler Funktion (56,9\%) als ausschließlich kausale Präpositionen (43,1\%). Im Gegensatz dazu verwendeten die niederländischen Studierenden die Präpositionen mit ausschließlich 
kausaler Bedeutung häufiger $(65,9 \%)$ als optional kausale $(34,1 \%)$. Die deutschen Studierenden benutzten im Vergleich zu den niederländischen Studierenden jedoch signifikant öfter kausale und optional kausale Präpositionen $(p<0,05)$. Auf Basis der prozentuellen Verteilung scheint das eher unlogisch zu sein, jedoch wurden von den deutschen Studierenden in absoluten Zahlen viel häufiger Präpositionen verwendet als von den niederländischen Studierenden. Dieser signifikante Unterschied hängt daher wahrscheinlich damit zusammen, dass die deutschen Studierenden im Allgemeinen häufiger Präpositionen verwendeten. Die Unterschiede sind in Abbildung 4 grafisch dargestellt.

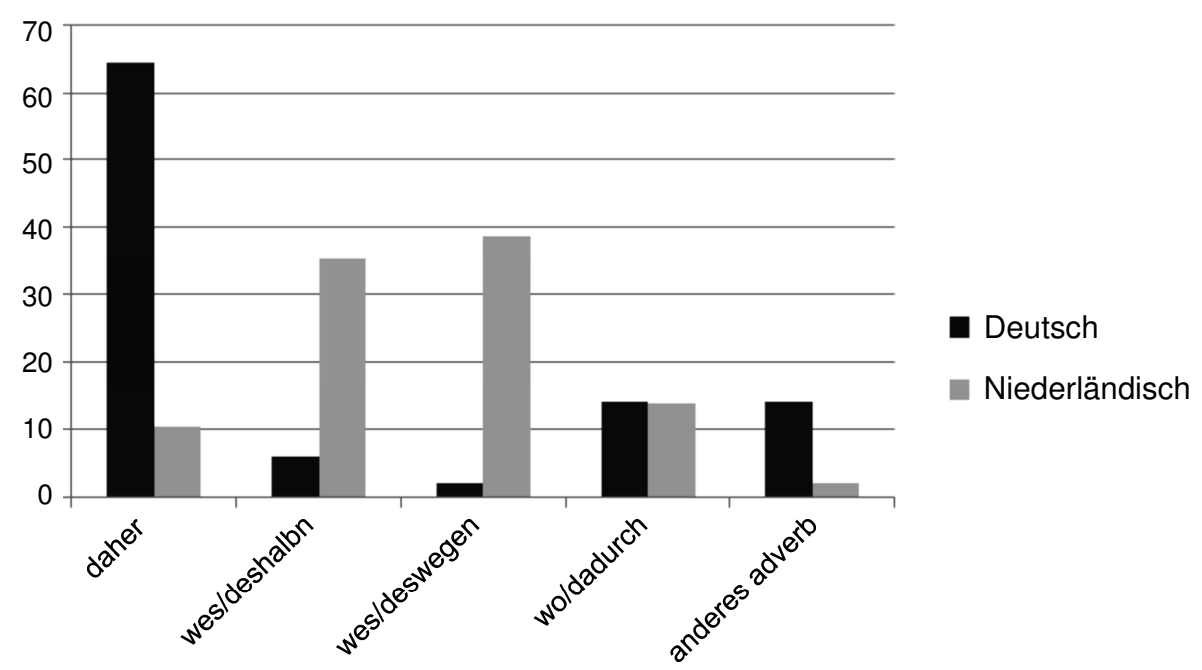

Abbildung 5. Grafische Darstellung der Unterschiede im Gebrauch der kausalen Adverbien zwischen den deutschen und den niederländischen Studierenden in Prozentzahlen

Ein letzter Unterschied auf Wortniveau findet sich bei den lexikalischen Mitteln. Es gibt einen Effekt der L1 auf die Verwendung der einzelnen Adverbien $\left(X^{2}(4)=207,306, p<0,001\right)$. Abbilung 5 zeigt die im L1- und L2-Korpus eingesetzten kausalen Adverbien. Auffallend ist, dass die deutschen Studierenden sich bei fast zwei Drittel der benutzen Adverbien für das Adverb ,daher' (62,4\%) entschieden haben. Die zweitgrößte Kategorie der Adverbien wird von,wo/dadurch' gebildet. Die Anzahl der Verwendungen von ,wes/deshalb' und ,wes/deswegen' im deutschen Korpus ist zu vernachlässigen. Die niederländischen Studierenden entschieden sich im Gegensatz zu den deutschen Studierenden nur in 10,3\% der Fälle, in denen 
sie ein Adverb benutzten, für ,daher'. Dies ist signifikant weniger häufig erfolgt als bei den deutschen Studierenden $(p<0,05)$. In der Mehrheit der Fälle, in denen sie ein Adverb verwendeten, entschieden die Niederländer sich gerade für die Adverbien, die von den deutschen Studierenden kaum benutzt wurden, nämlich ,wes/deshalb' (35,3\%) und ,wes/deswegen' $(38,7 \%)$, diese Adverbien wurden von den niederländischen Studierenden signifikant häufiger verwendet $(p<0,05)$. Nur im Gebrauch von,wo/dadurch' stimmten die beiden Gruppen in etwa überein $(p>0,05)$, vgl. Abbildung 5 .

\section{FAZIT: ANREGUNG ZUR UMGESTALTUNG VON LERNERGRAMMATIKEN (C1/C2)}

Auf Basis der dargestellten Ergebnisse kann festgehalten werden, dass es zwar Übereinstimmungen zwischen den deutschen und niederländischen Studierenden im Ausdruck von Kausalität gibt, dass sie sich aber im spezifischen Gebrauch der kausalen Ausdrucksmittel in ihren wissenschaftlichen Texten erheblich voneinander unterscheiden. So stimmten die deutschen und niederländischen Studierenden in dem Anteil, zu dem die Texte durchschnittlich aus Kausalität bestanden (deutsches Korpus: 0,70\%, niederländisches Korpus: 0,68\%), überein. Zudem stimmten auch die Verhältnisse zwischen den grammatischen, lexikalischen und anderen Mitteln in den beiden Korpora überein. Der Unterschied zwischen dem deutschen Korpus (51,9\% bzw. $47,9 \%$ bzw. 0,2\%) und dem niederländischen Korpus $(53,7 \%$ bzw. $46,1 \%$ bzw. 0,2\%) war nur sehr gering und nicht signifikant. Diese Feststellungen sprechen dafür, dass die von den niederländischen Studierenden geschriebenen Texte im großen Umfang eine zielsprachenadäquate Realisation von Kausalität im Deutschen zeigen, und dass die Studierenden sich in Übereinstimmung mit den deutschen Daten sowohl für grammatische als auch für lexikalische Mittel entscheiden, wenn sie Kausalität ausdrücken möchten. Die fortgeschrittenen niederländischen Lerner des Deutschen verfügen somit über eine angemessene wissenschaftliche Schreibkompetenz, um Kausalität, bezüglich des Anteils im Text und der Verteilung über grammatische und lexikalische Mittel, zielsprachenadäquat auszudrücken. Diese Kompetenz kann aus der L1 übernommen (in Übereinstimmung mit Mächler, 2012), oder von den Studierenden bereits erworben worden sein (in Übereinstimmung mit Mohammadi, 2009).

Wenn man die Ergebnisse jedoch detaillierter betrachtet, zeigen sich bei den beiden Studierendengruppen durchaus Unterschiede. Partizipialkonstruktionen wurden von beiden Gruppen vergleichsweise selten eingesetzt, wobei die niederländischen Studierenden diese fast gänzlich vernachlässig- 
ten und somit die deutschen Studierenden diese signifikant öfter gebrauchten. Partikeln, die in der Regel nicht zum wissenschaftlichen Sprachinventar gerechnet werden, wurden von den Deutschen kaum eingesetzt; die Niederländer benutzten diese Wortart signifikant häufiger. Ein weiterer auffälliger Unterschied zeigt sich darin, dass die deutschen Studierenden die übrigen Wortarten (Präpositionen, Nomina, Verben und Adverbien) ungefähr gleich häufig verwendeten, während es im niederländischen Korpus weniger Variation und größere Präferenzen für bestimmte Wortarten gab. Die signifikant häufigere Verwendung von Partikeln und Haupt- und Nebensätzen sowie die Variation in der Verwendung der Präpositionen, Nomina, Verben und Adverbien führen zu der Schlussfolgerung, dass der Gebrauch der verschiedenen Subkategorien der grammatischen und lexikalischen Mittel von den niederländischen Studierenden nicht-zielsprachenadäquat ist.

Eine Betrachtung der benutzten Wörter in einigen Subkategorien führt zu noch größeren Unterschieden zwischen den beiden Korpora. Für die Hauptund Nebensätze galt, dass die deutschen Studierenden eine deutliche Präferenz für die Subjunktion ,da' hatten. Diese wurde in ungefähr drei Viertel der Fälle, in denen ein Haupt- oder Nebensatzkonnektor benutzt wurde, verwendet. Im Gegensatz dazu wurde, weil' kaum verwendet. Im niederländischen Korpus wurde ,weil' jedoch am häufigsten verwendet (und damit signifikant öfter als von den Deutschen), die Subjunktion ,da' wurde zwar am zweithäufigsten benutzt, jedoch signifikant weniger oft als von den Deutschen. Des Weiteren fiel auf, dass die deutschen Studierenden sich öfter für andere, optional kausale Präpositionen entschieden, während die niederländischen Studierenden öfter kausale Präpositionen verwendeten. Schließlich gab es auch innerhalb der Subkategorie der Adverbien große Unterschiede. Während die deutschen Studierenden vor allem ,daher' verwendeten und kaum ,deshalb' und ,deswegen' einsetzten, sind Letztere beide gerade die Adverbien, die die niederländischen Studierenden präferierten. ,Daher' wurde von den Niederländern nur sehr selten und im Vergleich zum deutschen Korpus signifikant seltener verwendet. Die niederländischen Studierenden unterscheiden sich somit auf Wortniveau am auffälligsten von den deutschen Studierenden. Eine Analyse auf dieser Ebene zeigt, dass die Fremdsprachenlerner die jeweiligen Wörter nicht mit der Frequenz verwendeten, die dem deutschen Korpus zufolge zielsprachenadäquat gewesen wäre (z.B. ,da' und ,daher').

Interferenz könnte eine Ursache für die unterschiedlichen Verwendungen sein; wie u.a. Kántor-Faragó (2006) und Zufferey et al. (2015) gezeigt haben, können minimale Unterschiede in der Bedeutung und dem Gebrauch z.B. der Kausalkonjunktionen, zu nicht-zielsprachenadäquater Anwendung in der Fremdsprache führen. Bei der Durchführung dieser Studie wurde ein Vergleich zwischen niederländischen Lernern des Deutschen und L1-Schrei- 
bern des Deutschen gemacht, wobei die deutschen Daten als Referenzdaten galten, mit deren Hilfe die Zielsprachenadäquatheit überprüft wurde. $\mathrm{Ob}$ die gefundenen Unterschiede zwischen der niederländischen und deutschen Gruppe tatsächlich als Interferenz erklärt werden können, kann im Rahmen dieser Studie nicht definitiv bestätigt oder widerlegt werden, da entsprechende niederländische Vergleichdaten fehlen.

Eine andere Ursache der vorliegenden Ergebnisse kann in der Gestaltung des Fremdsprachenunterrichts, der u.a. von Grammatikdarstellungen in Lehrbüchern und Lernergrammatiken geprägt wird, gesehen werden. Die ,Grundgrammatik Deutsch' von Kars und Häussermann (1992) für Anfänger (A1-B1 nach GER) nennt z.B. bei den Konjunktionen, die einen Grund ausdrücken, ,weil' und ,da' (in der Reihenfolge) und bei der Erklärung der Wortarten ,deshalb' als typisches Adverb zur Beantwortung einer,WarumFrage'. Die ,deutsche Grammatik' von Engel (1988) für fortgeschrittene Lerner (B2+ nach GER) bespricht bei den Kausalangaben ,deshalb', ,darum', ,aus diesem Grund' und ,weil' und bietet erst in den jeweiligen Kapiteln zu den Konjunktionen und Adverbien eine vollständige, alphabetisch geordnete Liste an. Die Konnektoren, die von diesen Autoren zum Ausdruck von Kausalität hervorgehoben bzw. als Erstes eingeführt werden, sind im Allgemeinen diejenigen, die von den niederländischen Deutschlernern am häufigsten verwendet wurden, obwohl die Verwendung dieser Wörter dem deutschen Korpus zufolge für wissenschaftliche Texte nicht-zielsprachenadäquat ist. Eine geringe Fokussierung auf die tatsächlichen Möglichkeiten, Kausalität im Deutschen in bestimmten Textsorten auszudrücken, scheint somit dazu zu führen, dass Lerner des Deutschen einige zielsprachenadäquaten Ausdrucksweisen nicht kennen bzw. nicht im angemessenen Umfang einsetzen und sich auch sehr fortgeschrittene niederländische Lerner des Deutschen der Frequenz, in der zielsprachenadäquate Wörter eingesetzt werden, nicht bewusst sind.

Neben Interferenz als möglicher Grund für die im Rahmen dieser Studie gefundenen Unterschiede, dürfte somit auch die Präsentation sprachlicher Strukturen in Lehrbüchern und die Gestaltung der Lernergrammatiken von größerem Einfluss sein. Mehr Aufmerksamkeit bzw. eine Sensibilisierung für zielsprachenadäquate Ausdrucksweisen einer bestimmten Funktion, wie Kausalität, statt einer bestimmten Form, wie Nebensätze, könnte die fortgeschrittene fremdsprachliche Schreibkompetenz verbessern. Die ,Grammatik in Feldern' von Buscha et al. (1998) formt hierfür eine gute Basis und könnte zudem mit genaueren Angaben zum zielsprachenadäquaten Gebrauch (Wörter und Verhältnis) in bestimmten Textsorten ergänzt werden. Auch wenn eine solche Gestaltung sich für Anfängergrammatiken weniger eignen würde, könnte sie sich für fortgeschrittene Lerner durchaus lohnen. 


\section{LITERATURVERZEICHNIS}

Albert, R. (2008). Beurteilung der Qualität von Grammatiken - ein wichtiges Ausbildungsziel für DaF-Studierende. In: C. Chlosta / G. Leder / B. Kirscher (Hrsg.), Auf neuen Wegen. Deutsch als Fremdsprache in Forschung und Praxis. 35. Jahrestagung des Fachverbandes Deutsch als Fremdsprache 2007 an der Freien Universität Berlin (S. 93-108). Göttingen: Universitätsverlag Göttingen.

Blühdorn, H. (2006). Kausale Satzverknüpfungen im Deutschen. In: Pandaemonium germanicum, 10, S. 253-282.

Breindl, E. / Waßner, U. (2006). Syndese vs. Asyndese. Konnektoren und andere Wegweiser für die Interpretation semantischer Relationen in Texten. In: H. Blühdorn / E. Breindl / U. Waßner (Hrsg.), Text - Verstehen. Grammatik und darüber hinaus (S. 46-70). Berlin: De Gruyter.

Buscha, J. / Freudenberg-Findeisen, R. / Forstreuter, E. / Koch, H. / Kuntzsch, L. (1998). Grammatik in Feldern. Ein Lehr- und Übungsbuch für Fortgeschrittene. Ismaning: Verlag für Deutsch.

Duden (2009). Die Grammatik. Mannheim: Dudenverlag.

Einig, C. / Menne-El-Sawy, G. (2012). Problemfeld: Sprachliche Register in der Wissenschaftssprache. Info DaF, 39 (4), S. 385-404.

Engel, U. (1988). Deutsche Grammatik. Heidelberg: Julius Groos Verlag.

Helbig, G. / Buscha, J. (1991). Deutsche Grammatik: Ein Handbuch für den Ausländerunterricht. 14. Auflage. Berlin-München: Langenscheidt.

Jentges, S. (2011). Texte schrittweise verfassen... unter Berücksichtigung zielsprachenspezifischer Variation auf satzinitialer Position. In: D. Knorr / A. Nardi (Hrsg.), Fremdsprachliche Textkompetenz entwickeln (S. 237-248). Frankfurt u.a.: Peter Lang.

Jentges, S. (2012). „Grammatisch und semantisch korrekt - aber dennoch nicht perfekt!“ Schriftliche Textproduktionen niederländischer L2-Lernender des Deutschen. In: U. Preußer / N. Sennewald (Hrsg.), Literale Kompetenzentwicklung an der Hochschule (S. 235-244). Frankfurt u.a.: Peter Lang.

Jentges, S. (2016). „Die Holländer sollen mehr Deutsch reden“ - Textsortendidaktik im Germanistikstudium in den Niederlanden am Beispiel von Modalverbkonstruktionen. In: R. Freudenberg-Findeisen (Hrsg.), Auf dem Weg zu einer Textsortendidaktik. Linguistische Analysen und text(sorten)didaktische Bausteine nicht nur für den fremdsprachlichen Deutschunterricht (S. 201-218). Hildesheim: Georg Olms-Verlag.

Kántor-Faragó, M. (2006). Kausalkonjunktionen im Deutschen und im Niederländischen. KontrastivKorpuslinguistische Untersuchungen und sprachtypologisch-methodologische Überlegungen zum Problem der rückwärts kausalen Konjunktionen. Debrecen: Doktorarbeit Universität Debrecen.

Kars, J. / Häussermann, U. (1992). Grundgrammatik Deutsch. 4. Auflage. Frankfurt am Main: Verlag Moritz Diesterweg.

Laurentzen, C. (2016). Zielsprachenadäquate Ausdrücke von Kausalität: Da oder weil, daher oder deswegen? Zur Realisierung von Kausalität durch niederländische und deutsche Studierende in deutschsprachigen wissenschaftlichen Texten. Masterarbeit Radboud Universiteit Nijmegen. http://theses.ubn.ru.nl/ bitstream/handle/123456789/2427/Laurentzen,\%20C.M.J.\%204 186184.pdf?sequence=1 [16.05.2017].

Mächler, L. (2012). Erwerb des wissenschaftlichen Schreibens in der Fremdsprache Deutsch. Exemplarische Analyse von intertextuellen Prozeduren. Info DaF, 39(5), S. 519-539. 
Mohammadi, V. (2009). Transfer eigenkultureller Textnormen in die fremdsprachliche Textproduktion. In: D. Skiba (Hrsg.), Diskurs- und Textmuster: schulisch - universitär - kulturkontrastiv (S. 127-142). Frankfurt am Main: Peter Lang.

Smith, R. / Frawley, W. (1983). Conjunctive cohesion in four English genres. Text $\mathcal{E}$ Talk, 3/4, S. 347-374.

Ten Cate, A. / Lodder, H. / Kootte, A. (2013). Deutsche Grammatik: Eine kontrastiv deutschniederländische Beschreibung für den Fremdspracherwerb. Bussum: Uitgeverij Coutinho.

Zufferey, S. / Mak, W. / Sanders, T. (2015). A cross-linguistic perspective on the acquisition of causal connectives and relations. International review of pragmatics, 7, S. 22-39.

Received: 16.05.2017; revised: 9.06.2017 
